\title{
3D MORPHOLOGICAL MODELLING OF A RANDOM FIBROUS NETWORK
}

\author{
Charles PeyregA ${ }^{1}$, Dominique Jeulin ${ }^{1}$, Christine Delisée ${ }^{2}$ And Jérôme \\ MALVESTIO $^{2}$
}

${ }^{1}$ Centre de Morphologie Mathématique, Mathématiques et Systèmes, Mines ParisTech, 35 rue Saint Honoré, 77300 Fontainebleau, France, ${ }^{2}$ Unité des Sciences du Bois et des Biopolymères, 351, cours de la Libération 33405 Talence CEDEX, France

e-mail: charles.peyrega@mines-paristech.fr, dominique.jeulin@ensmp.fr, delisee@us2b.pierroton.inra.fr, malvestio@us2b.pierroton.inra.fr

(Accepted September 14, 2009)

\begin{abstract}
In the framework of the Silent Wall ANR project, the CMM and the US2B are associated in order to characterize and to model fibrous media studying 3D images acquired with an X-Ray tomograph used by the US2B. The device can make 3D images of maximal $2304^{3}$ voxels with resolutions in the range of $2 \mu \mathrm{m}$ to $15 \mu \mathrm{m}$. Using mathematical morphology, measurements on the 3D X-Ray CT images are used to characterize materials. For example measuring the covariance on these images of an acoustic insulating material made of wooden fibres highlights the isotropy of the fibres orientations in the longitudinal planes which are perpendicular to the compression Oz axis. Moreover, it is possible to extract other morphological properties from these image processing methods such as the size distribution either of the fibres or of the pores by estimating the morphological opening granulometry of the considered medium. Using the theory of random sets introduced by Georges Matheron in the early 1970's, the aim of this work is to model such a fibrous material by parametric random media in 3D according to the prior knowledge of its morphological properties (covariance, porosity, size distributions, etc.). A Boolean model of random cylinders in 3D stacked in planes parallel to each other and perpendicular to the $\mathrm{Oz}$ compression axis is first considered. The granulometry results provide gamma distributions for the radii of the fibres. In addition, a uniform distribution of the orientations is chosen, according to the experimental isotropy measurements in the longitudinal planes. Finally the third statistical factor is the length distribution of the fibres which can be fitted by an exponential distribution. Thus it is possible to estimate the validity of this model first by trying to fit the experimental transverse and longitudinal covariances of the pores with the theoretical ones taking into account the statistical distributions of the dimensions of the random cylinders. The second method to validate the model consists in comparing morphological measurements (density profiles, covariance, opening granulometry, tortuosity, specific surface area) processed on real and on simulated media.
\end{abstract}

Keywords: 3D images, Boolean model, fibrous media, mathematical morphology, random media.

\section{INTRODUCTION}

Fibrous materials are commonly used for thermal and acoustical insulating in buildings. The Silent Wall $\mathrm{ANR}^{1}$ project's objective is to build an acoustical insulating wall made of fibrous media, with innovating acoustical properties, developed in a context of environmental efficiency and competitiveness by using natural raw materials such as wood and other cellulose fibres. In this purpose, morphological measurements were performed (Peyrega et al., 2009a) to characterize the Thermisorel ${ }^{\mathrm{TM}}$, which is a wooden fibre board $100 \%$ naturally papermaking processed from recycled wood, used as a reference material in the Silent Wall project. This paper focuses on modelling the microstructure of such a fibrous material.

When 3D images were not available yet, 2D images from confocal microscope acquisitions were used to fit models of random fibrous media. The method proposed by Jeulin (2000), Castéra et al. (2000), Michaud et al. (2000) and Delisée et al. (2001) consists in generating a Boolean model of Poisson lines, dilated by spheres with a random radius (Matheron, 1967; Serra, 1982). This method is effective to simulate 3D stacks of very long fibres after identification from 2D projected images, when their length is large compared to the images. Modelling a fibrous medium with 3D Poisson lines was also made later by Schladitz et al. (2006) to study its acoustical properties. However, the dimensions of the fibres of Thermisorel ${ }^{\mathrm{TM}}$ in the present paper are finite compared to those of the sample (about $600 \times 600 \times 360$ voxels with resolutions ranging from $2 \mu \mathrm{m}$ to $15 \mu \mathrm{m}$ ). That is why a different model is proposed here.

\footnotetext{
${ }^{1}$ http://us2b.pierroton.inra.fr/Projets/Silent_Wall/description.htm
} 
As the fibres of Thermisorel ${ }^{\mathrm{TM}}$ have finite dimensions, they could be modelled by a Boolean model of random cylinders whose radii, lengths and orientations are estimated by the morphological measurements performed by Peyrega et al. (2009a). Moreover, these cylinders are stacked in the $x O y$ planes perpendicular to the axis of compression $\mathrm{Oz}$, according to the industrial papermaking process of this material. The covariance measurements of the fibres (Matheron, 1967; Serra, 1982) highlight the global anisotropy of the material, and the isotropy of their orientations in the $x O y$ planes. Thus, it seems to be realistic to simulate uniformly distributed orientations between 0 and $\pi$ in these planes. The opening granulometry of the fibres (Matheron, 1967; Serra, 1982) allows us to extract the distribution of their radii. Automatic morphological fibre analysis using 2D images with the $\mathrm{MorFi}^{\mathrm{TM}}$ system ${ }^{2}$ brings the distribution of their lengths out.

After having presented the 3D X-Ray CT images of Thermisorel ${ }^{\mathrm{TM}}$ used for the morphological characterization, the Boolean model of random cylinders will be introduced. Afterwards two methods to extract the distribution of the radii will be presented (the first one from the granulometry, and the second one from a minimization of the mean square error using the transverse covariance of the external pores). At last an estimation of the distribution of the lengths will be introduced.

\section{D IMAGES OF THERMISOREL}

The 3D images are obtained with the US2B XRay tomograph, with resolutions equal to $2 \mu \mathrm{m}, 5 \mu \mathrm{m}$, $9.36 \mu \mathrm{m}$ and $15 \mu \mathrm{m}$ per voxel, depending on the wanted observation scale. The grey level images are segmented by manual thresholding, after smoothing the noise by a low-pass filter (Fig. 1). Several structures are observable in the Thermisorel ${ }^{\mathrm{TM}}$ (Fig. $2 a)$. The fibrous phase is composed of isolated fibres and of clusters, the sticks. The external porosity and the internal one, i.e., the lumens into the fibres where the sap flows in the tree, compose the porous phase.

With a $9.36 \mu \mathrm{m}$ resolution, the lumens are not observable, but for $5 \mu \mathrm{m}$ they should be filled in order to isolate the external porosity. An algorithm to fill the lumens is proposed by Lux (2005). It consists in the succession of morphological operations on the segmented image. The final result is represented in Fig. $2 b$ on an image acquired in Grenoble on the beamline ID 19 of the European Synchrotron
Radiation Facility (ESRF) with a resolution of $5 \mu \mathrm{m} / \mathrm{voxel}$.

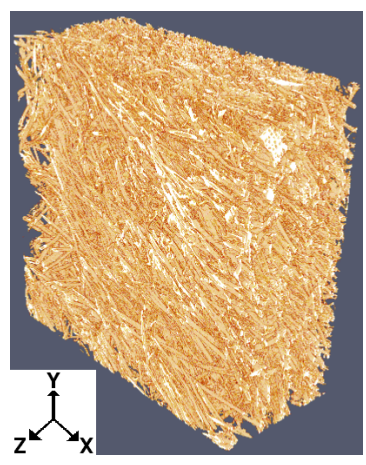

(a)

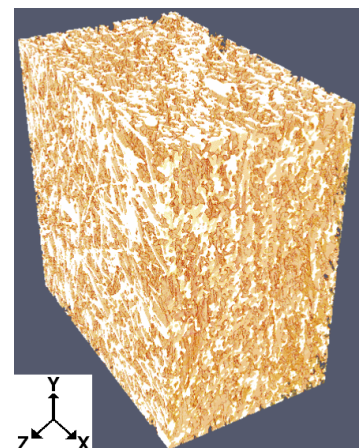

(b)
Fig. 1. Binarized X-Ray CT images of Thermisorel ${ }^{\mathrm{T}}$; (a) Source: ESRF; resolution: $5 \mu \mathrm{m} /$ voxel; dimensions: $5.9 \mathrm{~mm} \times 5.9 \mathrm{~mm} \times 1.68 \mathrm{~mm}$, (b) Source: US2B; resolution: $9.36 \mu \mathrm{m} /$ voxel; dimensions: $5.6 \mathrm{~mm} \times 5.6 \mathrm{~mm} \times 3.4 \mathrm{~mm}$.

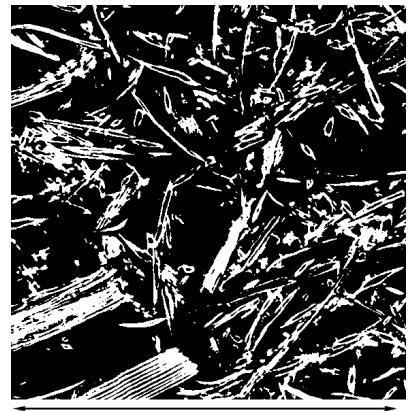

$2.9 \mathrm{~mm}$

(a)

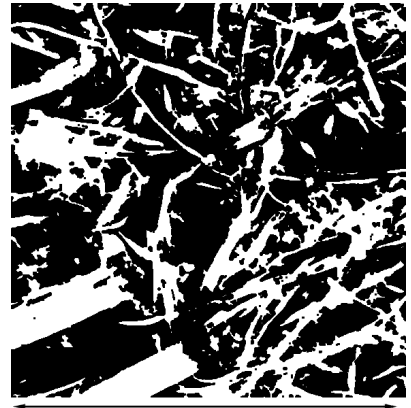

$2.9 \mathrm{~mm}$

(b)
Fig. 2. Lumen filling of Thermisore ${ }^{\mathrm{T}} \mathrm{M}$ (resolution: $5 \mu \mathrm{m} /$ voxel; source: ESRF ID19). (a) Segmented 2D slice of Thermisore $I^{\mathrm{T}}$ in the $x \mathrm{Oy}$ plane, (b) Filled lumens.

As the production process of Thermisorel ${ }^{\mathrm{TM}}$ is a papermaking one, the fibrous mat is compressed along the $O z$ axis. The fibres are thus isotropically oriented in the $x O y$ planes perpendicular to $O z$. This is observable on the measurements of the covariance $C(h)$ of the fibres (Peyrega et al., 2009a), showing identical covariances in the $x O y$ planes, whatever the orientation of the vector $\vec{h}$, which is a consequence of the transverse isotropy. However, the fibrous medium is anisotropic in the other directions of 3D space, since the correlation length in the $O z$ direction is shorter than in the $x O y$ plane.

\footnotetext{
${ }^{2}$ http://cerig.efpg.inpg.fr/dossier/EFPG-innovations/page10.htm
} 


\section{BOOLEAN MODEL OF RANDOM CYLINDERS}

\section{THE BOOLEAN MODEL}

In order to model a Thermisorel ${ }^{\mathrm{TM}}$-like fibrous medium, with fibres having finite lengths, the Boolean model with random cylinders as primary grains seems to be suitable. The Boolean model was introduced by Matheron (1967) and used by Jeulin (2000) in a modelling of random textures for materials. The first step consists in generating a Poisson point process.

Random primary grains $A^{\prime}$ are implanted on Poisson points $x_{k}$ with the intensity $\Theta$ i.e., the average number of generated points per unit volume. The overlap of these grains is possible. Let $A$ be the random set generated by the grains (Eq. 1),

$$
A=\bigcup_{x_{k}} A_{x_{k}}^{\prime} .
$$

\section{REMINDER ON THEORETICAL PROPERTIES OF THE BOOLEAN MODEL}

$A$ is the set of fibres with $p=P(x \in A)$. The probability $Q(K)$ for a compact set $K$ to be included in the set $A^{c}$ (with $q=P\left(x \in A^{c}\right)=1-p$ ) is given by Matheron (1967) and Serra (1982) as a function of the erosion $\ominus$ and dilation $\oplus$ operations in Eq. 2 and Eq. 3 . Let $\overline{\mu_{n}}$ be the average Lebesgue measure (average volume in 3D), and $\breve{K}=\{-x, x \in K\}$ be the transposed set of $\mathrm{K}$.

$$
\begin{aligned}
& Q(K)=P\left(K \subset A^{c}\right)=P\left(x \in A^{c} \ominus \check{K}\right), \\
& Q(K)=\mathrm{e}^{-\Theta \overline{\mu_{n}}\left(A^{\prime} \oplus \check{K}\right)}=q^{\frac{\bar{n}_{n}\left(A^{\prime} \oplus \check{K}\right)}{\bar{n}_{n}\left(A^{\prime}\right)}} .
\end{aligned}
$$

The covariance $(Q(h))$, linear erosions $(Q(l))$, and erosions by balls $(Q(B(r)))$ are particular cases for $K$ of the expression given in Eq. 2 and Eq. 3.

\section{Covariance $\mathrm{Q}(\mathrm{h})$}

$A$ is the set of fibres. Let $Q(h)$ (Eq. 4) be the covariance of the porous medium, i.e., of the set $A^{c}$. The Eq. 5 gives the theoretical expressions of $Q(h)$ for the Boolean model. Let $K(h)$ be the geometrical covariogram of the grain $A^{\prime}, K(h)=\overline{\mu_{n}}\left(A^{\prime} \cap A_{-h}^{\prime}\right)$. The normalized covariogram $r(h)$ is defined by $r(h)=$ $K(h) / K(0)$, with $K(0)=\overline{\mu_{n}}\left(A^{\prime}\right)$,

$$
\begin{gathered}
Q(h)=P\left(x \in A^{c}, x+h \in A^{c}\right), \\
Q_{\text {BooleanModel }}(h)=q^{2} \mathrm{e}^{\Theta(K(h))}=q^{2-r(h)} .
\end{gathered}
$$

From Eq. 5 it is then possible to deduce the porosity $q$ (Eq. 6), which leads to the intensity $\Theta$ of the Poisson point process from which the Boolean model has been generated (Eq. 7).

$$
\begin{aligned}
q & =\mathrm{e}^{-\Theta \overline{\mu_{n}}\left(A^{\prime}\right)}, \\
\Theta & =\frac{-\ln (q)}{\overline{\mu_{n}}\left(A^{\prime}\right)} .
\end{aligned}
$$

\section{Linear erosions $\mathrm{Q}(\mathrm{I})$}

Considering the porous phase, the theoretical expression $Q(l)$ is given by Eq. 8 for a random convex grain $A^{\prime}$ in which we consider $r^{\prime}(0)=(\mathrm{d} r / \mathrm{d} h)_{h=0}$.

$$
Q_{\text {BooleanModel }}(l)=\mathrm{e}^{-\Theta \overline{\mu_{n}}\left(A^{\prime} \oplus l\right)}=q^{1-l r^{\prime}(0)} .
$$

\section{Erosions by balls $\mathrm{Q}(\mathrm{B}(\mathrm{r}))$}

The probability for a ball with radius $r, B(r)$ to be included in the pores is given by Eq. 9:

$$
Q_{\text {BooleanModel }}(r)=\mathrm{e}^{-\Theta \overline{\mu_{n}}\left(A^{\prime} \oplus B(r)\right)} .
$$

In the case of convex grains, like random cylinders, the $\overline{\mu_{n}}\left(A^{\prime} \oplus B(r)\right)$ can be expanded according to the Steiner's formula involving the Minkowski's functionals of $A^{\prime}$ and of $B(r)$. It is therefore a polynomial of degree 3 in $r$. According to the Steiner's formula, it is possible to write Eq. 10 which implies Eq. 11. Let us consider $V(K)$ the volume of a compact set $K, A(K)$ its surface area, and $M(K)$ its integral mean curvature, with $\bar{R}=E[R]$ and $\bar{L}=E[L]$.

$$
\begin{aligned}
\overline{\mu_{n}}\left(A^{\prime} \oplus B(r)\right)= & V\left(A^{\prime}\right)+\frac{M(B(r)) S\left(A^{\prime}\right)}{4 \pi} \\
& +\frac{M\left(A^{\prime}\right) S(B(r))}{4 \pi}+V(B(r)), \\
\overline{\mu_{n}}\left(A^{\prime} \oplus B(r)\right)= & \frac{4}{3} \pi r^{3}+\pi(\bar{L}+\pi \bar{R}) r^{2} \\
& +[2 \pi \bar{R}(\bar{R}+\bar{L})] r+\pi E\left[R^{2}\right] \bar{L} .
\end{aligned}
$$

These expressions, as well as the exponential behavior of $Q_{\text {BooleanModel }}(l)$ are used as to test the assumption of a Boolean model with convex grains.

\section{TEST OF THE VALIDITY OF A BOOLEAN MODEL FOR THE FIBROUS NETWORK}

In order to validate the assumption of a Boolean model of random cylinders to simulate a Thermisorel ${ }^{\mathrm{TM}}$-like material, several morphological characteristics of the fibrous network have been compared to the theoretical results. As a first step, these measurements are the linear erosions $Q(l)$ 
(Fig. 3 with a logarithmic scale) and the erosions by rhombicuboctaedra $Q(B(r))$ (Fig. 4 with a logarithmic scale, and Fig. 7).

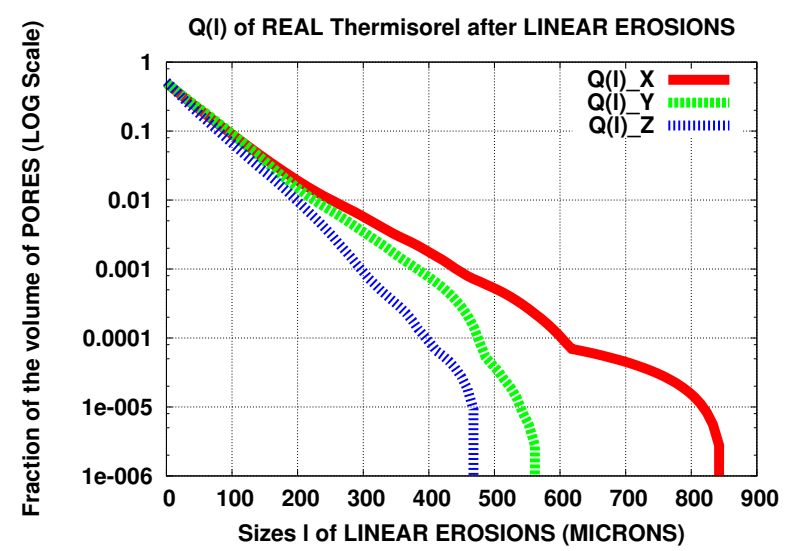

Fig. 3. Linear erosions of Thermisore $\mathrm{I}^{\mathrm{M}}$ in the $O x, O y$ and $\mathrm{Oz}$ directions, shown in logarithmic scale (source: sample Fig. $1 b$ ).

In the $\mathrm{Ox}$ and $\mathrm{Oy}$ longitudinal directions $\log (Q(l))$ cannot be fitted exactly by a straight line (Fig. 3). This result is due to the fact that fibres are not strictly convex. They should be cylinders with some slight bending, which modifies the behavior of $\log (Q(l))$ in the directions of the $x O y$ planes. In the $\mathrm{Oz}$ direction, $\log (Q(l))$ can be fitted by a straight line. However, we will neglect these points for those fibres, and use the 'perfect' cylinders assumption.

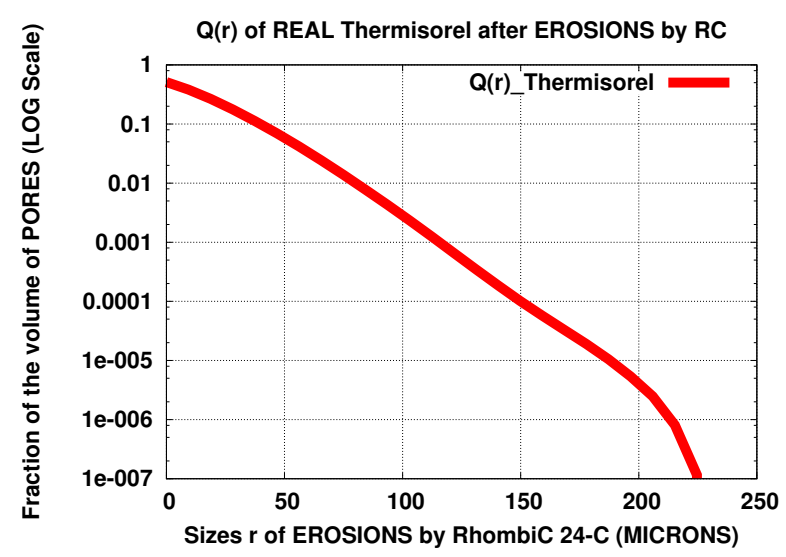

Fig. 4. Erosions by rhombicuboctaedra B(r) of Thermisore ${ }^{\mathrm{T}}$, shown in logarithmic scale (source: sample Fig. 1b).

In Fig. 4, the experimental curve $\log (Q(B(r)))$ $(B(r)$ being a ball of radius $r$ ) should be fitted by a theoretical polynomial of degree 3 in $r$ (Eq. 11).

\section{BOOLEAN MODEL OF RANDOM CYLINDERS WITH A TRANSVERSE ISOTROPY}

We consider now as primary grains a population of cylinders with a random radius $R$ and a random length $L$. A given grain is completely known from these two characteristics and from its orientation. With respect to the already mentioned transverse isotropy of the network, we will consider fibres orthogonal to the $O z$ axis, and with a uniform distribution of orientations in the $x O y$ planes. In this context, the most general random fibre model would require the knowledge of the bivariate distribution of $R$ and $L, f(r, l)$. For simplification, and in absence of simultaneous data on the same fibres, we will assume here that these two random variables are independent.

Knowing the distributions for $R$ and for $L$, it is possible to compute the theoretical covariance in the transverse direction (along $O z$ ), or in any horizontal direction in the $x O y$ planes (longitudinal covariance). The input of the observed size distributions enables us to check the validity of the model, by comparison of the measured and calculated covariances. Alternatively, we can also fit the parameters of the distribution from the experimental covariances, by a least squares minimization.

Consider first the transverse covariance of the pores, $Q_{Z}(h)$. From Eq. 5, it is expressed as a function of the transverse reduced covariogram of the cylindrical fibre, $r_{Z_{\text {Cylinder }}}(h)$.

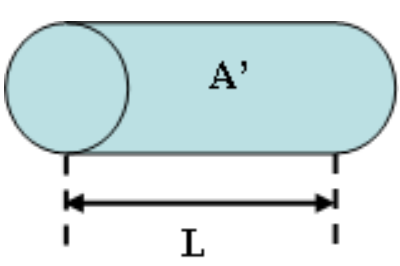

(a)

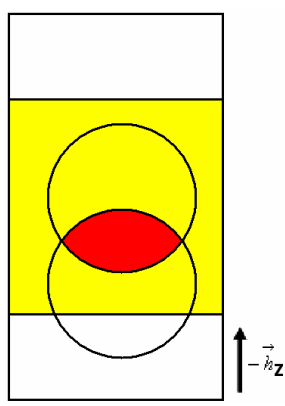

(b)
Fig. 5. Transverse geometrical covariogram of the cylinder. (a) Cylindrical primary grain A', (b) Geometrical covariogram of the disc (red area).

Let $f_{1}(r)$ be the distribution of the random radii of the fibres. The transverse geometrical covariogram of a fibre is given as a function of the average length $\overline{L_{\text {fibres }}}$ and of the average geometrical covariogram of a population of discs with a random radius $R$ following the distribution $f_{1}(r)$ by $K_{Z_{\text {Cylinder }}}(h)=\overline{L_{\text {fibres }}} \overline{K_{Z_{\text {Disc }}}}(h)$. Therefore we have: 


$$
r_{Z_{\text {Cylinder }}}(h)=\frac{\overline{K_{Z_{\text {Disc }}}}(h)}{\overline{K_{Z_{\text {Disc }}}}(0)} .
$$

The normalized transverse geometrical covariogram of the fibres does not depend on their lengths, as a result of the independence between $R$ and $L$. In Eq. $12, \overline{K_{Z_{\text {Disc }}}}(h)$ is deduced from the geometrical covariogram of a disc $K_{Z_{\text {Disc }}}(h, r)$ (Fig. 5) and from $f_{1}(r)$. We have, if $0 \leq h \leq 2 r$ (if $h>2 r$, $\left.K_{Z_{\text {Disc }}}(h, r)=0\right)$ :

$$
\begin{aligned}
& K_{Z_{\text {Disc }}}(h, r)=2 r^{2}\left(\arccos \left(\frac{h}{2 r}\right)\right. \\
& \left.-\frac{h}{2 r} \sqrt{1-\left(\frac{h}{2 r}\right)^{2}}\right) \text {, }
\end{aligned}
$$

and

$$
\overline{K_{Z_{\text {Disc }}}}(h)=\int_{h / 2}^{+\infty} K_{Z_{\text {Disc }}}(h, r) f_{1}(r) \mathrm{d} r .
$$

Consider now the longitudinal covariance $Q_{x O y}(h)$, given for a vector $\vec{h}$ in the horizontal xOy planes (due to the transverse isotropy this vector can be considered as parallel to the $O x$ axis). Noting $f_{2}(l)$ the distribution function of the random length $L$, the average longitudinal geometrical covariogram of the fibre $\overline{K_{x} O_{y_{\mathrm{Cyl}}}}(h)$ is given by Eq. 16 which is illustrated in Fig. 6.

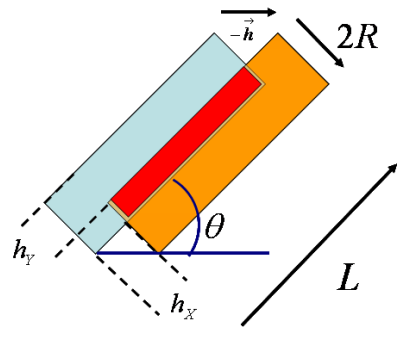

(a)

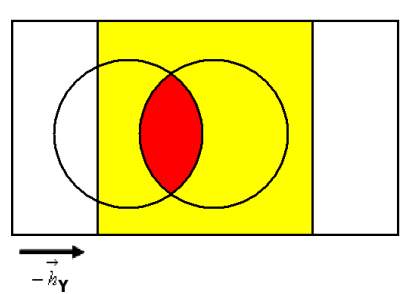

(b)
Fig. 6. Longitudinal geometrical covariogram of the cylinder (red volume). (a) Geometrical covariogram, xOy projection, (b) Geometrical covariogram, yOz projection.

The red volume in the Fig. 6 corresponds to the geometrical covariogram of the cylinder and is defined by the Eq. 15, for $h_{X} \leq l$ (for $h_{X}>l, K_{x O y_{\text {Cylinder }}}(h, \theta)=$ 0 ),

$$
K_{x O y_{\text {Cylinder }}}\left(h_{X}, h_{Y}, l\right)=\left(l-h_{X}\right) \overline{K_{Z_{\text {Disc }}}}\left(h_{Y}\right) .
$$

Considering $h_{X}=h \cos (\theta), h_{Y}=h \sin (\theta)$ and the uniform distribution of the orientations $\theta$ of the fibres it is then possible to write the Eq. 16, $f_{2}(l)$ being the distribution of the random length $L$ of the fibres,

$$
\begin{aligned}
& \overline{K_{x O y_{C y l}}}(h)=\int_{(h \sin \theta) / 2}^{+\infty} K_{Z_{\text {Disc }}}(h, r) f_{1}(r) \mathrm{d} r \\
& \cdot \frac{1}{\pi} \int_{0}^{\pi} \int_{h \cos \theta}^{+\infty}(l-h \cos \theta) f_{2}(l) \mathrm{d} l \mathrm{~d} \theta .
\end{aligned}
$$

The theoretical expression of the longitudinal covariance of the pores is obtained by using the Eq. 17 into the Eq. 5,

$$
r_{x O y_{\mathrm{Cylinder}}}(h)=\frac{\overline{K_{x O y_{\mathrm{Cyl}}}(h)}}{\overline{K_{x O y_{\mathrm{Cyl}}}(0)}} .
$$

\section{IDENTIFICATION OF THE PARAMETERS OF THE MODEL AND EXPERIMENTAL RESULTS}

After having defined the theoretical properties of the Boolean model, the objective is to fit them with the experimental measurements.

\section{DISTRIBUTION FUNCTIONS}

Knowing the porosity $q$, the first step consists in estimating the intensity $\Theta$ of the Poisson point process from which the Boolean model originates. In the case of cylinders as primary grains $A^{\prime}$, with radii $R$ and lengths $L$, both independent random variables, we can write the Eq. 18 in the Eq. 7:

$$
\overline{\mu_{n}}\left(A^{\prime}\right)=\bar{V}\left(A^{\prime}\right)=\pi E\left[R^{2}\right] E[L] .
$$

In the second step the distribution functions of $R$ and $L$ are estimated. The opening granulometry by rhombicuboctaedra (Fig. 7 and Fig. 9) gives access to a 'volume' weighted distributions of the radii of the fibres (Matheron, 1967; Serra, 1982).

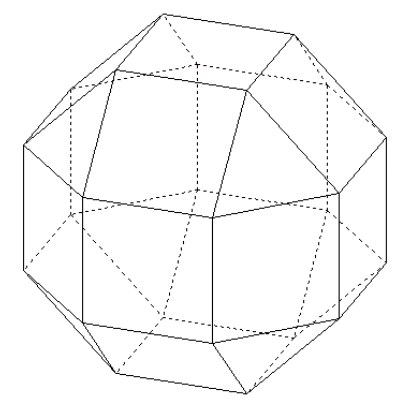

Fig. 7. Rhombicuboctaedron.

The morphological measurements presented by Peyrega et al. (2009a) show that the distribution in 'volume' of the radii of the fibres $g_{\alpha_{1}, b_{1}}(r)$ can be fitted 
by a gamma distribution (Eq. 19) with parameters $\alpha_{1}=$ $E[R]^{2} / \operatorname{Var}(R)$ and $b_{1}=E[R] / \operatorname{Var}(R)$,

$$
\begin{aligned}
g_{\alpha, b}(r) & =\frac{b^{\alpha}}{\Gamma(\alpha)} r^{\alpha-1} \mathrm{e}^{-b r}, \\
\Gamma(\alpha) & =\int_{0}^{+\infty} t^{\alpha-1} \mathrm{e}^{-t} \mathrm{~d} t .
\end{aligned}
$$

Moreover it is possible to link 'volume' $g(r)$, and 'number' $f_{1}(r)$ weighted granulometries as gamma distributions with the following formula (Eq. 21, Peyrega et al., 2009a). Then we can write the Eq. 22 in the Eq. 18,

$$
\begin{gathered}
f_{1}(r)=g_{\left[\alpha_{1}-2, b_{1}\right]}(r), \\
E\left[R^{2}\right]=\frac{\alpha(1+\alpha)}{b^{2}}=\frac{\left[\alpha_{1}-2\right]\left(1+\left[\alpha_{1}-2\right]\right)}{b_{1}^{2}} .
\end{gathered}
$$

For the Thermisorel ${ }^{\mathrm{TM}}$ material, we obtain the following parameters: $\alpha_{1}=6.53$ and $b_{1}=0.164 \mu \mathrm{m}^{-1}$.

It appears that the distribution of $L$ follows approximately an exponential distribution $f_{2}(l)$ as obtained with the MorFi ${ }^{\mathrm{TM}}$ system. Therefore it comes for the 'number' distribution $f_{2}(l)$ :

$$
f_{2}(l)=\frac{1}{E[L]} \mathrm{e}^{-l / E[L]} .
$$

For the Thermisorel ${ }^{\mathrm{TM}}$ material, we obtain $E[L]=$ $1654 \mu \mathrm{m}$. In both cases, the quality of the fit can be measured by the mean square error (MSE).

\section{FITTING THE TRANSVERSE COVARIANCE}

The Fig. 8 represents the transverse covariance $Q_{Z}(h)$ of the pores. The thick plain red curve gives the experimental covariance. We can notice that this covariance reaches its theoretical sill without oscillations, which is required for a Boolean model. It shows that in the present case a hard-core point process for the location of the centres of fibres, instead of a Poisson point process, would be irrelevant. The blue middle dashed curve is the theoretical prediction of the covariance obtained with the fitted gamma distribution of the radii of the fibres with parameters $\alpha=4.53$ and $b=0.164 \mu \mathrm{m}^{-1}$, which gives a MSE $=1.16 \times 10^{-5}$ for $Q_{Z}(h)$.

Alternatively, we can fit the parameters of the distribution by minimizing the mean square error between the experimental and the theoretical $Q_{Z}(h)$ curves in the linear zone, i.e., for $0 \leq h \leq 56.16 \mu \mathrm{m}$. This method consists in initializing both $\alpha$ and $b$ parameters and then to make them vary around the values estimated from the granulometry. The theoretical covariance is then calculated using the corresponding gamma distribution $f$ in 'number'. The couple of parameters $\left(\alpha_{\mathrm{MSE}}, b_{\mathrm{MSE}}\right)$ minimizing this error is then chosen.

Here, for $f_{1}$ we obtain $\alpha_{\mathrm{MSE}}=4.98$ and $b_{\mathrm{MSE}}=$ $0.159 \mu \mathrm{m}^{-1}$, for a minimized MSE $=0.84 \times 10^{-5}$ for $Q_{Z}(h)$, which gives parameters close to the direct estimation obtained from the opening size distribution. The Fig. 9 shows the corresponding gamma distributions fitting the experimental 'volume' granulometry of the fibres. Both distributions are very close to each other and have quite similar scale parameters $b$. This shows that the two estimations of the parameters (from the granulometry and from the transverse covariance) are consistent, which validates the model.

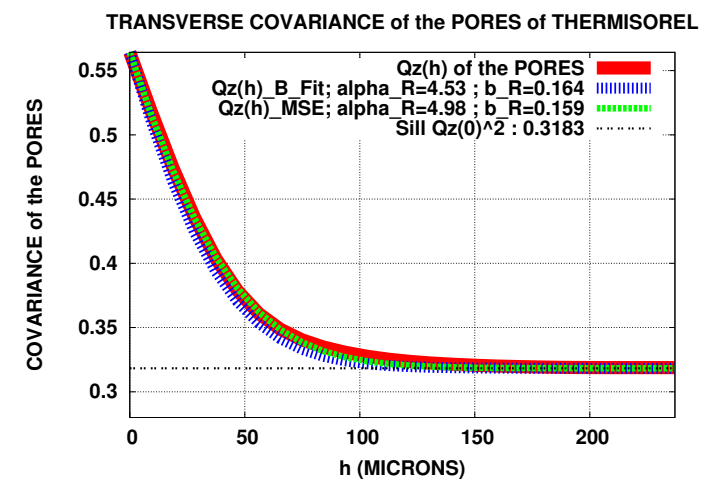

Fig. 8. Transverse covariance $Q_{Z}(h)$ of Thermisorel ${ }^{\mathrm{T}}$. Thick plain red: experimental data; middle dashed blue: theoretical values with a data-estimated gamma distribution for ' $r$ '; thin dashed green: theoretical values with a MSE-estimated gamma distribution.

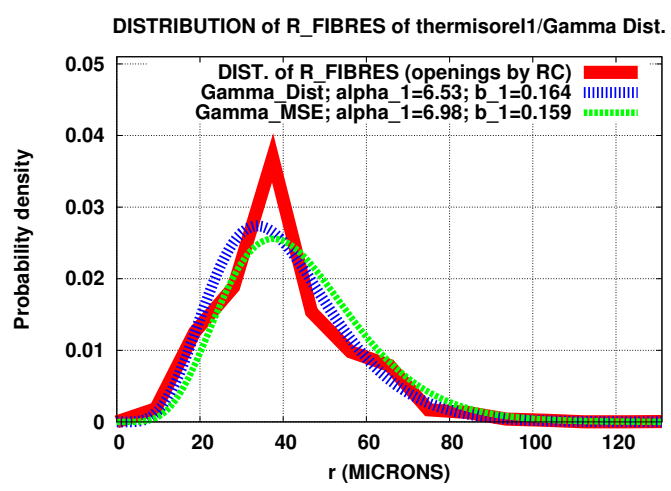

Fig. 9. Volume weighted distribution of the radii of the fibres of Thermisore ${ }^{\mathrm{TM}}$ and fitting by gamma distributions. Thick plain red: experimental data; middle dashed blue: data-estimated gamma distribution for ' $r$ '; thin dashed green: gamma distribution with MSE-estimated parameters. 


\section{FITTING THE LONGITUDINAL COVARIANCE}

Considering the longitudinal covariance in the Ox direction, the Fig. 10 shows that the Boolean model of random cylinders correctly fits the experimental data as well with a MSE $=0.27 \times 10^{-5}$ for $Q_{X}(h)$ with parameters $\alpha=4.53$ and $b=0.164 \mu \mathrm{m}^{-1}$ for $f_{1}$ and $E[L]=1654 \mu \mathrm{m}$ for $f_{2}$.

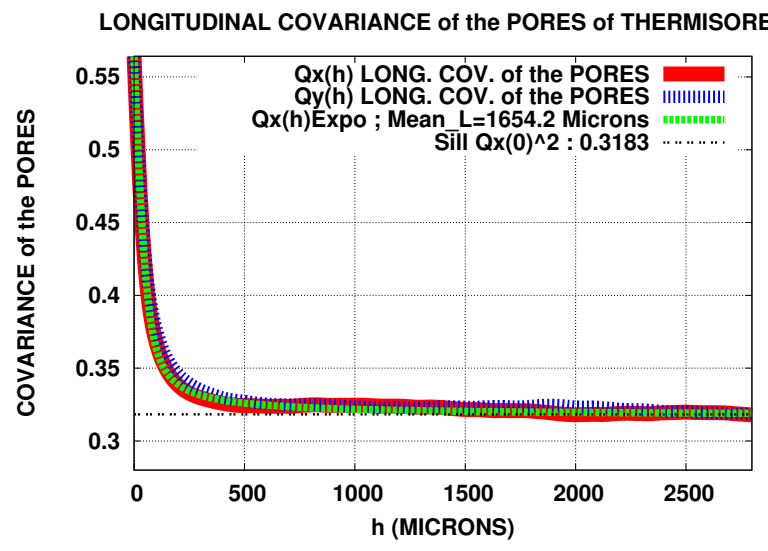

Fig. 10. Longitudinal covariances $Q_{X}(h)$ and $Q_{Y}(h)$ of Thermisorel ${ }^{\mathrm{T}}$. Thick plain red and medium dashed blue: experimental data; thin dashed green: theoretical values with data-estimated gamma and exponential distributions respectively for $R$ and $L$.

\section{EXAMPLE OF 3D SIMULATION}

For illustration a 3D simulation of the Thermisorel $^{\mathrm{TM}}$ fibrous network is shown in Fig. 11 and Fig. 12 to be compared respectively to Fig. 1 and Fig. 13. This simulation was processed in 4 minutes for about 18000 fibres with a desktop computer Intel ${ }^{\mathrm{TM}}$ Core 2 Extreme X6800 $2.93 \mathrm{GHz}$. The input parameters of this simulation are those estimated from Fig. $1 \mathrm{~b}$ (porosity $q=0.5642$; gamma distribution of the radii of the cylinders $\alpha=4.53$ and $b=0.164 \mu \mathrm{m}^{-1}$; mean length $E[L]=1654 \mu \mathrm{m}$ ).

As a consequence of the Boolean model, rectangles can be observed on the $2 \mathrm{D}$ slices in the $\mathrm{xOy}$ projection (Fig. 12a), and sections of ellipses in the zOy planes (Fig. 12b).

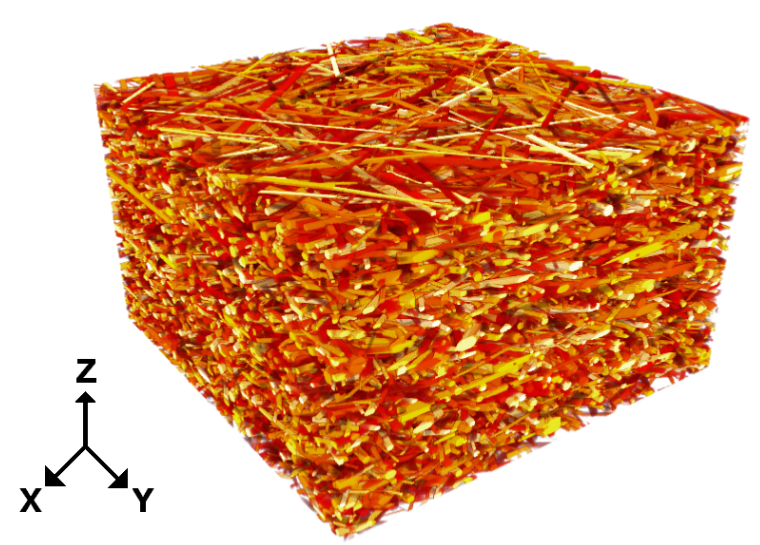

Fig. 11. Boolean model of cylinders (Dimensions: $5.6 \mathrm{~mm} \times 5.6 \mathrm{~mm} \times 3.4 \mathrm{~mm}$. Resolution: $9.36 \mu \mathrm{m} /$ voxel.)

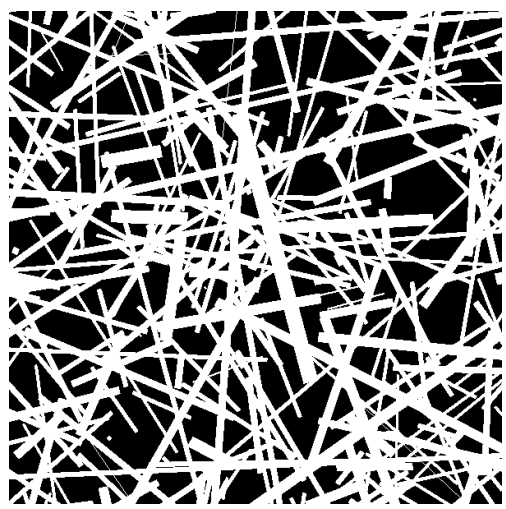

(a)

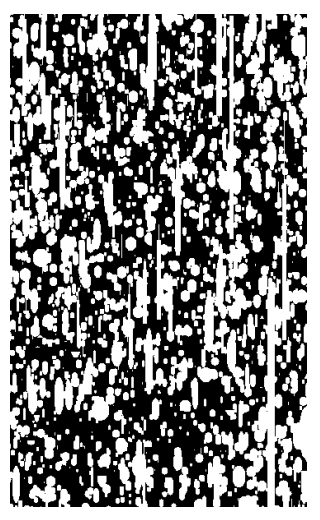

(b)
Fig. 12. Boolean model of cylinders (2D slices of Fig. 11). (a) Transverse xOy projection, (b) longitudinal zOy projection.

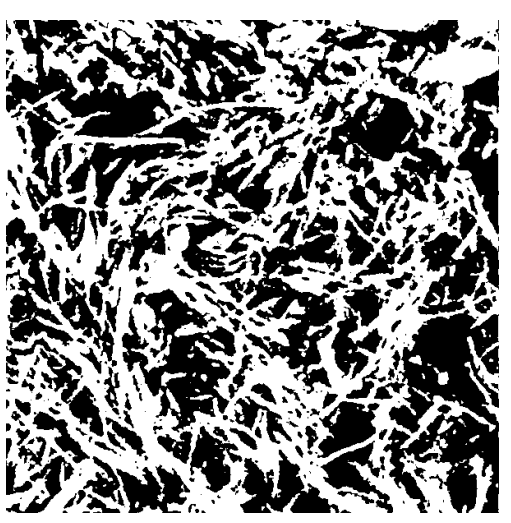

(a)

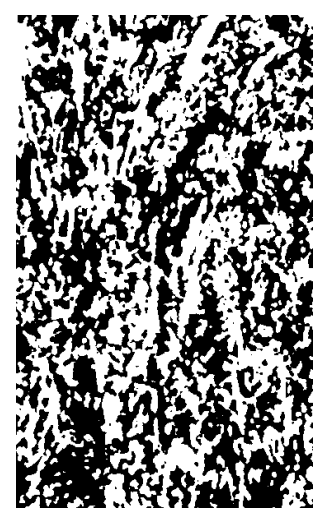

(b)
Fig. 13. Real Thermisore ${ }^{\mathrm{TM}}(2 \mathrm{D}$ slices of Fig. $1 b)$. (a) Transverse $x \mathrm{Oy}$ projection, (b) longitudinal zOy projection. 


\section{COMPARISON OF MORPHOLOGICAL MEASUREMENTS}

In order to validate the Boolean model of random cylinders, morphological measurements have been processed on both real (Fig. 1b) and simulated (Fig. 11) Thermisorel ${ }^{\mathrm{TM}}$.

\section{DENSITY PROFILES}

The porosity of the simulated fibrous material is equal to $56.30 \%$ and is consistent with the experimental porosity equal to $56.42 \%$. Moreover, the area fraction profiles of fibres in the $\mathrm{Ox}, \mathrm{Oy}$ and $\mathrm{Oz}$ directions are homogeneous for both media (Figs. 14 and 15).

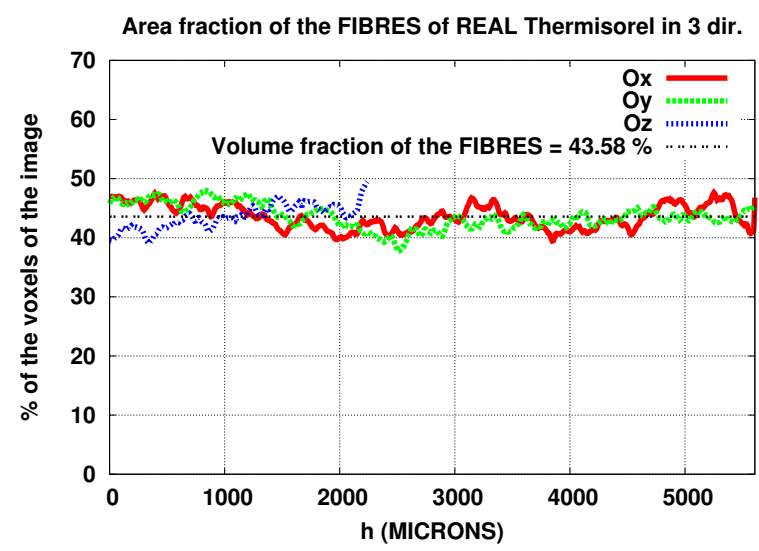

Fig. 14. Profiles of area fraction of real fibres in the $O x, O y$ and $O z$ directions.

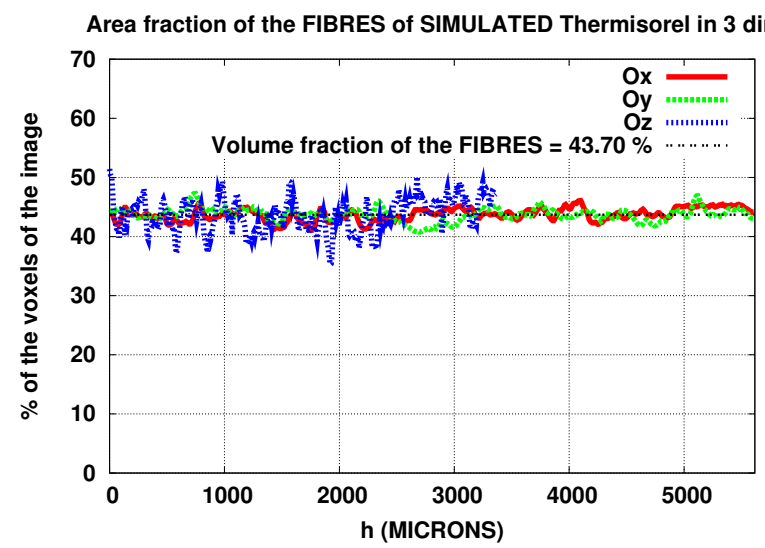

Fig. 15. Profiles of area fraction of simulated cylinders in the $O x, O y$ and $O z$ directions.

\section{COVARIANCE}

\section{Transverse covariance}

As shown in Fig. 16, the transverse covariances of real fibres and simulated cylinders (in the $\mathrm{Oz}$ direction) are almost superimposed. Thus both media have transverse characteristic lengths of the same order.

TRANS. COV. of the FIBRES of REAL/SIM. Thermisorel

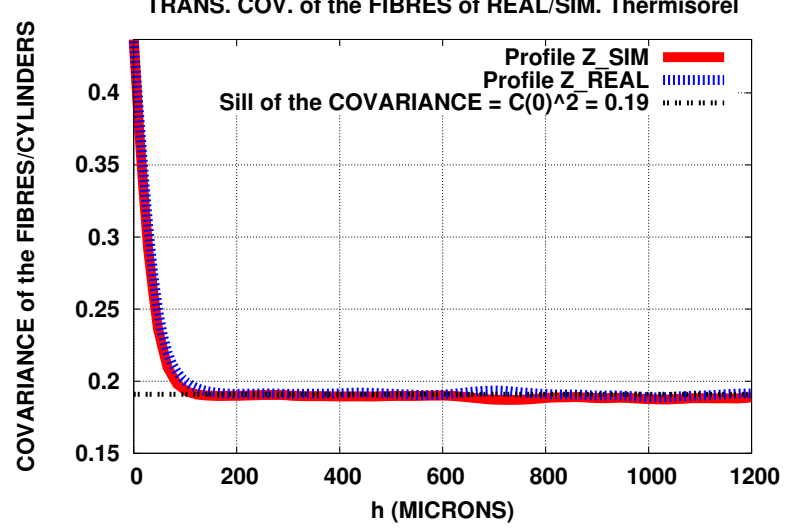

Fig. 16. Transverse covariance of fibres and simulated cylinders.

\section{Longitudinal covariance}

Concerning the longitudinal covariances of real fibres and simulated cylinders (in the $\mathrm{xOy}$ planes), the 4 curves in Fig. 17 are almost superimposed as well. Therefore both media have longitudinal characteristic lengths of the same order, with fibres and cylinders isotropically oriented in the $\mathrm{xOy}$ planes, since the covariances in the $\mathrm{Ox}$ and $\mathrm{Oy}$ directions are practically identical for both materials.

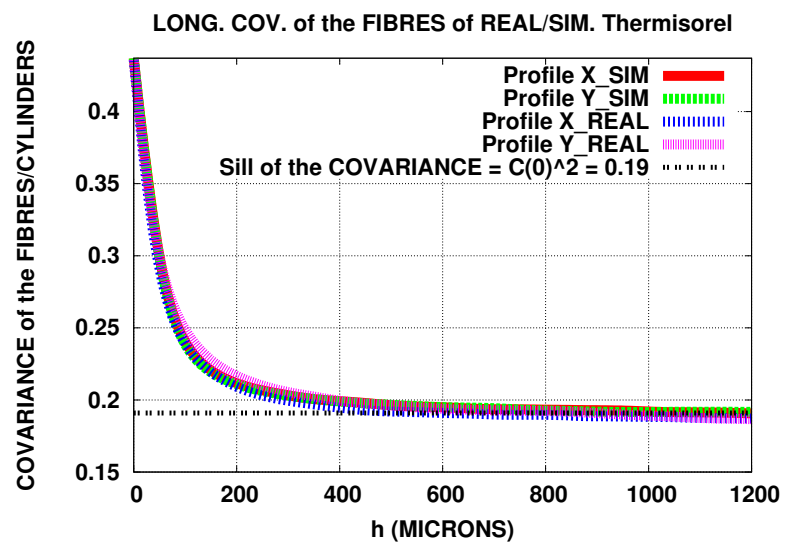

Fig. 17. Longitudinal covariance of fibres and simulated cylinders. 


\section{OPENING GRANULOMETRY}

The Fig. 18 and Fig. 19 represent the volume weighted opening granulometry respectively of the fibres and of the pores of real Thermisorel ${ }^{\mathrm{TM}}$. The openings are processed in 3D with rhombicuboctaedra as structuring elements. For both figures the corresponding fitted gamma distributions are plotted as well, with $\alpha=E[R]^{2} / \operatorname{Var}(R)$ and $b=E[R] / \operatorname{Var}(R)$.

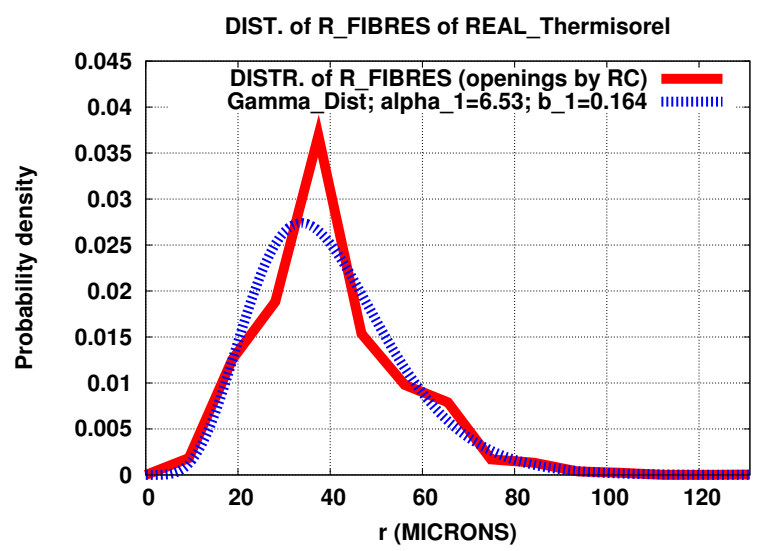

Fig. 18. Volume weighted opening granulometry of real fibres.

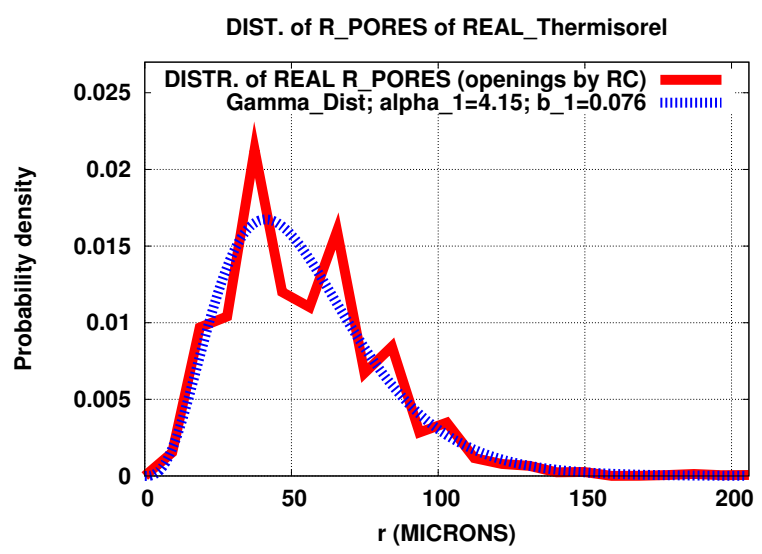

Fig. 19. Volume weighted opening granulometry of real pores.

The volume opening granulometries of the simulated cylinders and of the simulated pores (respectively Fig. 20 and Fig. 21) are slightly different from those of real Thermisorel ${ }^{\mathrm{TM}}$. Concerning the real fibres and the simulated cylinders, both fitted gamma distributions have quite similar parameters (difference of about 0.1 for $\alpha$ and difference of about $0.01 \mu \mathrm{m}^{-1}$ for $b$ ). Concerning the porous media, both fitted gamma distributions are different from each other (Figs. 19 and 21), but there is no theoretical requirement to use here gamma distributions. However both red curves have similar evolutions and the same local maxima in $r=37.44 \mu \mathrm{m}$ and $r=65.52 \mu \mathrm{m}$, and the same local minima in $r=28.08 \mu \mathrm{m}, r=46.8 \mu \mathrm{m}$ and $r=56.16 \mu \mathrm{m}$. These results validate the method used in this study which consists in processing the 3D opening granulometry directly on the real global fibrous medium to deduce the 'number' distribution of the radii of the fibres, without extracting them individually.

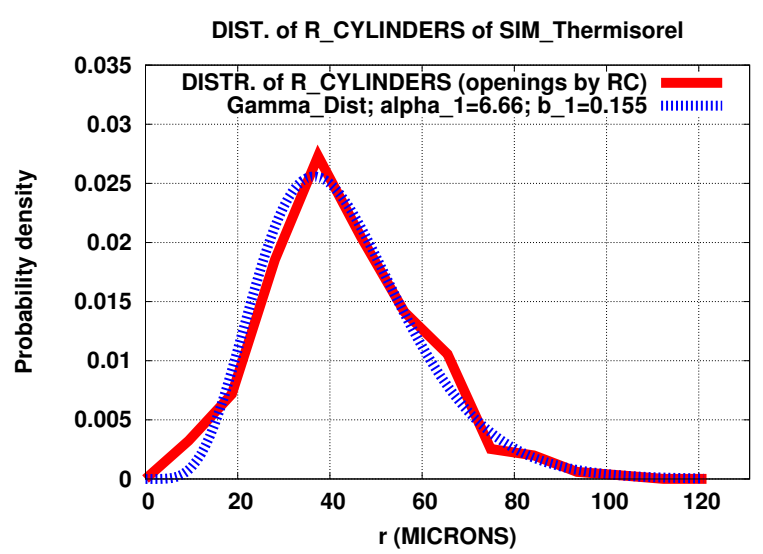

Fig. 20. Volume weighted opening granulometry of simulated cylinders.

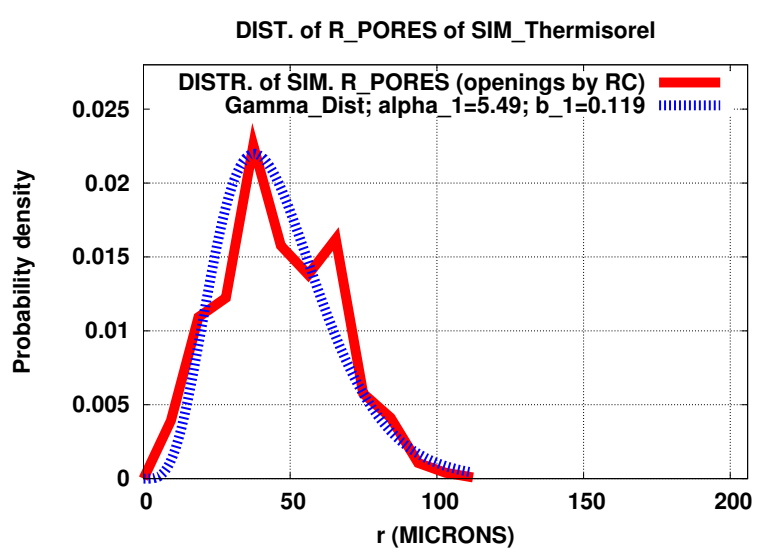

Fig. 21. Volume weighted opening granulometry of simulated pores.

\section{MORPHOLOGICAL TORTUOSITY}

The next characteristic to be compared is the morphological tortuosity, which corresponds to the ratio between the geodesic and the Euclidian distances between two parallel faces of the image according to a direction of propagation. The geodesic distance is processed either in the fibrous or in the porous phase. The minimum tortuosity is then equal to 1 by definition. The method to estimate it from 3D image processing is described by Peyrega et al. (2009a) and uses the algorithm proposed by Decker et al. (1998), which is coupled with a Fast Marching method 
(Sethian, 1996; Petres et al., 2005) to estimate the geodesic lengths of the paths in the media.

\section{In the fibrous media}

In the fibrous media, both Fig. 22 and Fig. 23 show a common trend. On the one hand the histograms of tortuosities in the transverse Ox and Oy directions are almost superimposed because of the isotropy of the orientations of the fibres and cylinders in the $\mathrm{xOy}$ planes. On the other hand, this implies lower mean tortuosities in every direction in these planes than in the $\mathrm{Oz}$ direction where higher tortuosities are reached.

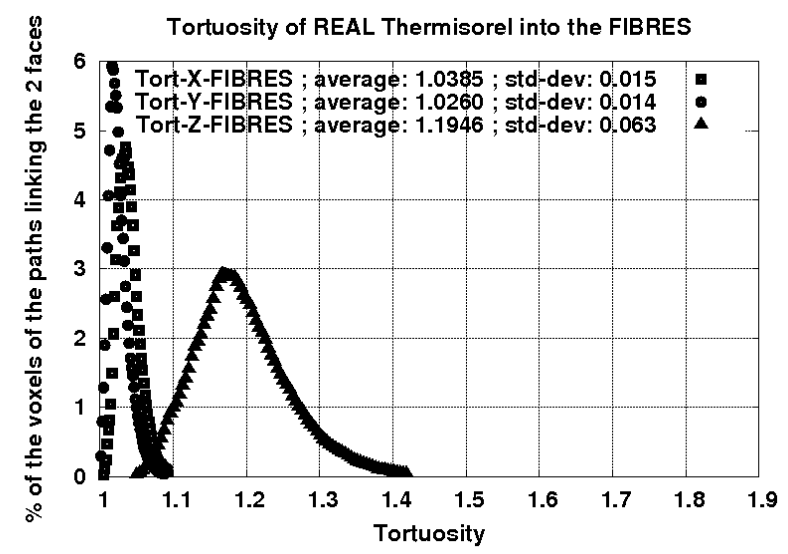

Fig. 22. Morphological tortuosity of real fibres in the $\mathrm{Ox}$, $\mathrm{Oy}$ and $\mathrm{Oz}$ directions.

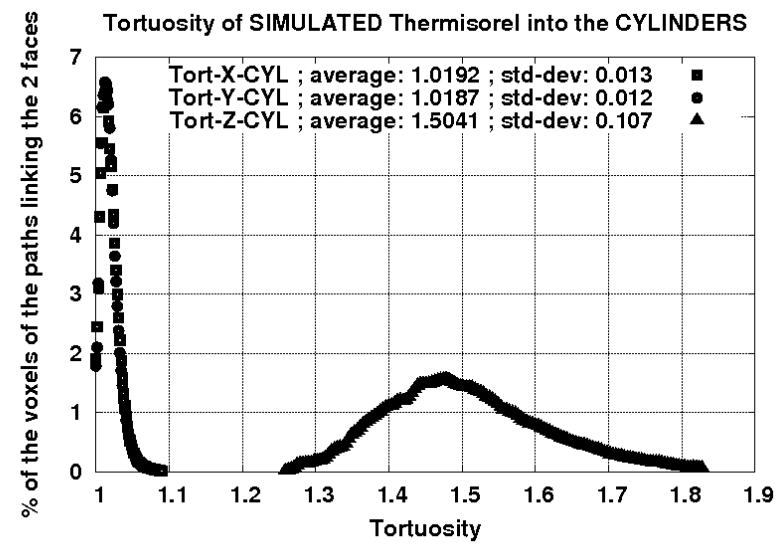

Fig. 23. Morphological tortuosity of simulated cylinders in the $O x, O y$ and $O z$ directions.

The transverse tortuosities of cylinders have mean values and standard deviations relatively similar to those of real fibres. However in the $\mathrm{Oz}$ direction, the real fibres are less tortuous, because they are not strictly straight and not strictly oriented in the xOy planes contrarily to the simulated ones. They are slightly curved.

\section{In the porous media}

The tortuosities in both materials are globally lower and close to 1 in the pores (Figs. 24 and 25), which proves that the pores are connected by straight paths, which will have an influence on the general acoustic behavior of the material.

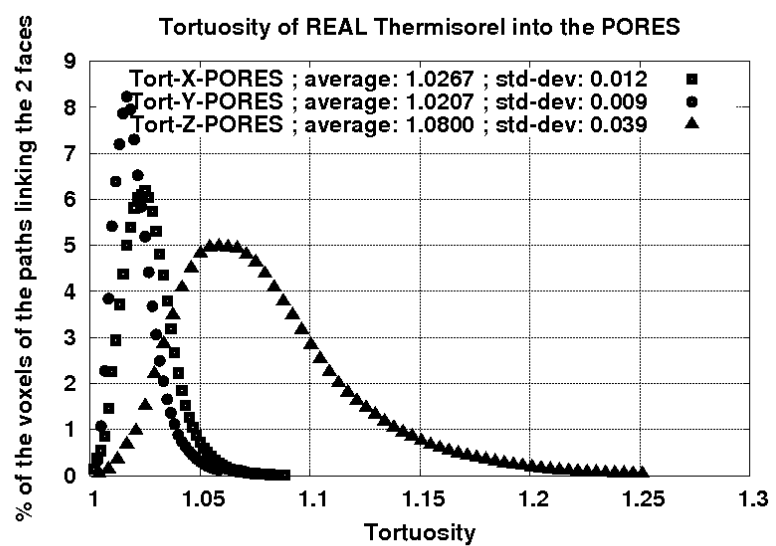

Fig. 24. Morphological tortuosity of real pores in the $\mathrm{Ox}$, $\mathrm{Oy}$ and $\mathrm{Oz}$ directions.

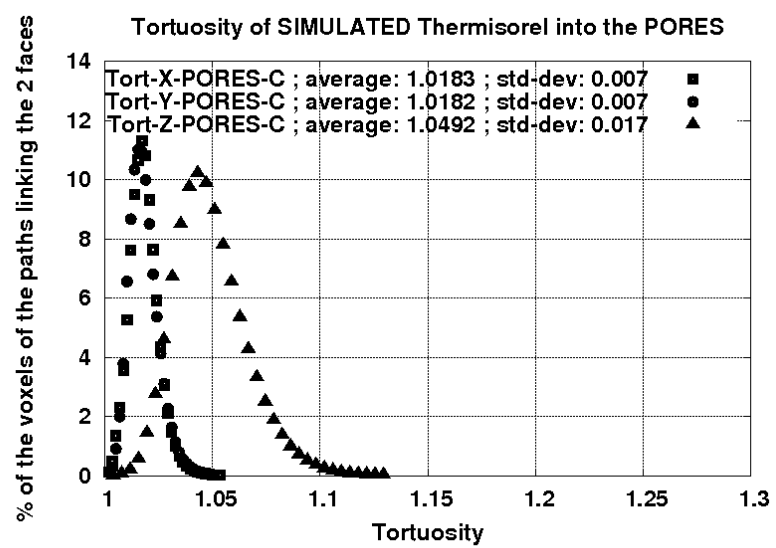

Fig. 25. Morphological tortuosity of simulated pores in the $\mathrm{Ox}, \mathrm{Oy}$ and $\mathrm{Oz}$ directions.

Like for the fibrous media, the tortuosities of the porous media according to $\mathrm{Ox}$ and $\mathrm{Oy}$ are lower than in the $\mathrm{Oz}$ direction and are similar for both real and simulated media. Moreover both histograms according to Ox and Oy in the simulated pores (Fig. 25) are quite superimposed because of transverse isotropy. Contrarily to the fibrous media, the tortuosities according to $\mathrm{Oz}$ in the real pores have mean values higher than those in the simulated material.

\section{SPECIFIC SURFACE AREA}

The specific surface area is the ratio between the contact surface between fibrous and porous media and the volume of the sample. This parameter has a direct 
influence on the thermal and acoustical heat transfer. It has been estimated by the Crofton formula. It is equal to $0.016 \mu \mathrm{m}^{-1}$ for real Thermisore $\mathrm{I}^{\mathrm{TM}}$ and equal to $0.017 \mu \mathrm{m}^{-1}$ for simulated cylinders. Both values are of the same order.

\section{IMPROVEMENT OF THE MODEL}

The first morphological measurements processed on this Boolean model of random cylinders seem to validate this method to build artificial Thermisorel ${ }^{\mathrm{TM}}$ like fibrous networks. However, as shown in the previous part, the tortuosity in the $\mathrm{Oz}$ direction, do not correctly fit the real material. This was in part explained by the fact that real fibres are not strictly oriented in the xOy planes. In spherical coordinates (Fig. 26), this means that their orientations $\phi$ are not strictly equal to $\frac{\pi}{2}$. On the contrary, they are randomly distributed around $\frac{\pi}{2}$ because of the compression of the sample.

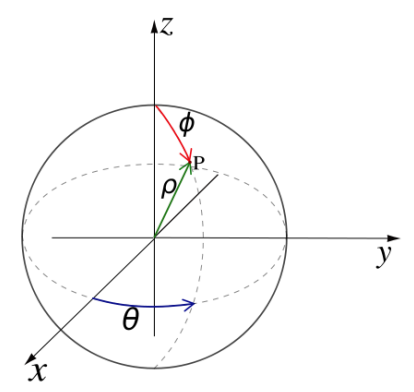

Fig. 26. Spherical coordinates.

\section{INTRODUCING A DISTRIBUTION OF ORIENTATIONS IN 3D}

In order to simulate these random orientations $\phi$, we use the distribution proposed by Schladitz et al. (2006) which is explicited in Eq. 24, with $\theta \in[0,2 \pi[$, and $\phi \in[0, \pi[$. We can notice that this distribution of orientations is independent from $\theta$. The anisotropy parameter $\beta$ is equal to 1 for isotropically distributed cylinders in $3 \mathrm{D}$. In order to simulate a compression of them along the $\mathrm{Oz}$ axis, $\beta$ is increased. Thus the previous model with cylinders oriented only in the $\mathrm{xOy}$ planes has a very high $\beta$,

$$
p(\theta, \phi)=\frac{1}{4 \pi} \frac{\beta \sin (\phi)}{\left[1+\left(\beta^{2}-1\right) \cos ^{2}(\phi)\right]^{3 / 2}} .
$$

Thus Fig. 27a and Fig. 27b respectively represent the probability density and the distribution functions of the distribution in Eq. 24 for $\beta=1,3$, and 10.

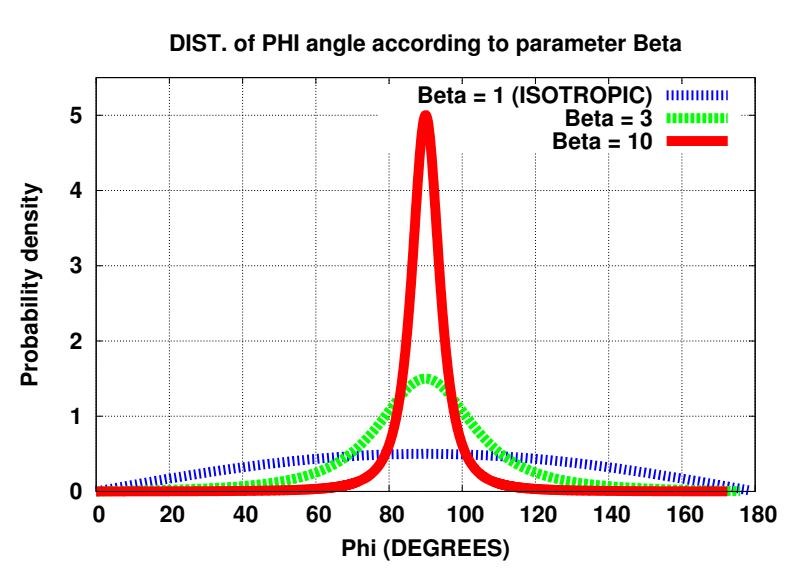

(a)

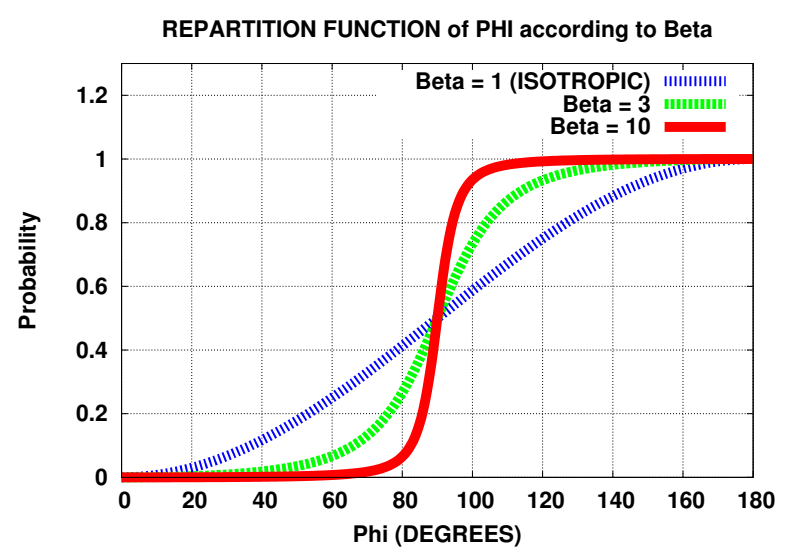

(b)

Fig. 27. Distribution of orientations $\phi$ in $3 D$ for $\beta=1,3$ and 10. (a) Probability density function, (b) distribution function.

\section{MORPHOLOGICAL MEASUREMENTS ON THE IMPROVED MODEL}

An improved sample having the same parameters than Fig. 11 and having a $\beta$ parameter equal to 10 is studied in this part. The same measurements than in the previous section have been processed on this sample. The density profiles, the covariance and the opening granulometry give similar results than for the sample on Fig. 11. The specific surface area is the same as well.

The only difference between both simulations lies in the tortuosities of the cylinders. As shown in Fig. 28 the histogram of the tortuosities of the simulated cylinders in the $\mathrm{Oz}$ direction is closer from the one of the real material (Fig. 22) than the previous model is (Fig. 23). However, we can notice that this new model is not improved for the tortuosities of the simulated pores according to Oz, (Fig. 29), which do not correctly fit the histogram of the real pores in the same 
direction (Fig. 24). Finally this modified version of the model still correctly fits the real Thermisorel ${ }^{\mathrm{TM}}$ for the tortuosities in the Ox and Oy directions whatever the medium considered (cylinders or simulated pores).

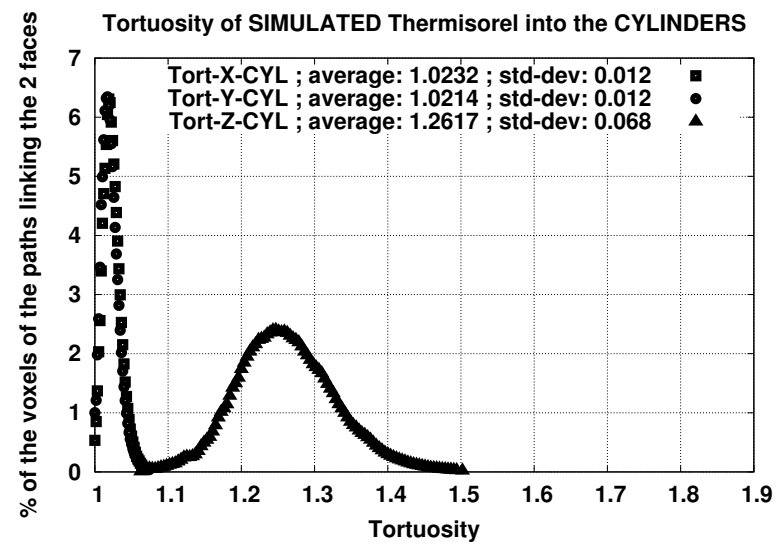

Fig. 28. Morphological tortuosity of simulated cylinders in the $O x, O y$ and $O z$ directions for the improved model with $\beta=10$.

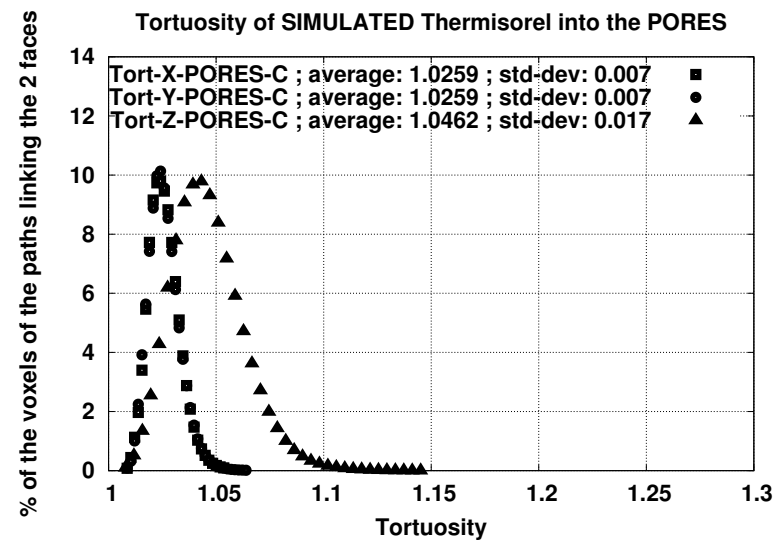

Fig. 29. Morphological tortuosity of simulated pores in the $O x, O y$ and $O z$ directions for the improved model with $\beta=10$.

\section{CONCLUSION}

It was shown that a Boolean model of random cylinders could describe properly a 3D fibrous network. In order to validate this assumption, it was necessary to fit the experimental measurements with the theoretical expressions of morphological parameters of the pores such as the transverse and longitudinal covariances.

The second step consisted in comparing morphological measurements of real and simulated media. The area fraction of fibres and cylinders are homogeneous for both materials. Moreover their transverse and longitudinal covariances have similar evolutions and characteristic lengths. The opening granulometries of the fibres and of the cylinders can be fitted by gamma distributions with similar parameters. The morphological tortuosities in the Ox and $\mathrm{Oy}$ directions of fibrous and porous media for both materials have quite the same histograms. However the tortuosities in the $\mathrm{Oz}$ direction are different for both materials whatever the phase considered, which can be explained by the geometrical differences between the cylinders and the real fibres, which are curved and not strictly oriented in the $\mathrm{xOy}$ planes. The last parameter which validates the model is the specific surface area which presents similar values for real and simulated media.

In order to improve the model, a distribution of orientations of the cylinders in 3D has been used. The morphological characteristics of this new material such as the density profiles, the covariance, the opening granulometry, the specific surface area, and the morphological tortuosities according to $\mathrm{Ox}$ and $\mathrm{Oy}$, still correctly fit the real Thermisorel ${ }^{\mathrm{TM}}$. Moreover the tortuosities of the cylinders in the $\mathrm{Oz}$ direction are closer from the histogram of the real material than the one of the previous model is. However there are not any improvement concerning the tortuosities of the simulated pores according to $\mathrm{Oz}$.

In a next step, the simulations will be used as input in the prediction of the acoustic properties of fibrous networks, with the aim to find microstructures with an optimized physical behavior.

\section{ACKNOWLEDGEMENTS}

This article is an extended version of a communication (Peyrega et al., 2009b) to the $10^{\text {th }}$ European Congress of Stereology and Image Analysis (ECS10, 2009 June 22-26; Milan, Italy).

\section{REFERENCES}

Castéra P, Jeulin D, Bos F, Michaud F, Delisée C (2000). On the role of microstructural morphology on the macroscopic properties of cellulosic fibrous materials. In: Proc Int Conf Wood Wood Fiber Compos, 2000 Apr 13-15; Stuttgart, Germany, 481-92.

Decker L, Jeulin D, Tovena I (1998). 3D morphological analysis of the connectivity of a porous medium. Acta Stereol 17(1): 107-12.

Delisée C, Jeulin D, Michaud F (2001). Caractérisation morphologique et porosité en 3D de matériaux fibreux cellulosiques. Compt Rend Acad Sci II B-Mec 329: 179-85.

Jeulin D (2000). Random texture models for materials structures. Stat Comput 10: 121-31. 
Lux J (2005). Comportement thermique macroscopique de milieux fibreux réels anisotropes; étude basée sur l'analyse d'images tridimensionnelles. PhD Thesis, Univ Bordeaux I, $n^{\circ} 3034$.

Matheron G (1967). Éléments pour une théorie des milieux poreux. Paris: Masson.

Michaud F, Jeulin D, Delisée C, Bos F (2000). On the morphological analysis and porosity of cellulosic fibrous materials. Compt Rend 12th Journ Natl Compos, 2000 Nov 15-17; Cachan, France, 449-58.

Petres C, Pailhas Y, Evans J, Petillot Y, Lane D (2005). Underwater Path Planning Using Fast Marching Algorithms. In: Proc IEEE Oceans 2005 Europe Conferences, 2005 June 20-23; Brest, France, 2: 814-9.

Peyrega C, Jeulin D, Delisée C, Malvestio J (2009a). 3D morphological characterization of fibrous media. Adv
Eng Mat, submitted.

Peyrega C, Jeulin D, Delisée C, Malvestio J (2009b). 3D morphological modelling of a random fibrous network. In: Capasso V et al., Ed., Proc 10th Eur Congr Stereol Image Anal (ECS10), 2009 June 22-26; Milan, Italy, 125-30.

Schladitz K, Peters S, Reinel-Bitzer D, Wiegmann A, Ohser J (2006). Design of acoustic trim based on geometric modeling and flow simulation for non-woven. Comput Mat Sci 38: 56-66.

Serra J (1982). Image analysis and mathematical morphology. London: Academic Press.

Sethian JA (1996). Level Set Methods. Evolving Interfaces in Geometry, Fluid Mechanics, Computer Vision, and Materials Science. Cambridge: Cambridge University Press, 81-95. 